injection as visualised with immunofluorescence microscopy. Induction of proteinuria occurred only after the transfer of HEL-specific B cells. In contrast, the presence of HEL in the GBM did not induce proteinuria, nor did the transfer of HELspecific $B$ cells in the absence of HEL injection. The proteinuria is associated with increased regions of podocyte effacement as visualised on transmission electron microscopy as compared to control mice. Additionally, the absence of periglomerular infiltrates on $\mathrm{H} \& \mathrm{E}$ stained sections were noted in both proteinuric and control mice. HEL-specific B cells demonstrated evidence of in vivo activation as they can be found in the spleen with their CFSE diluted. Finally, there is an absence of anti-HEL antibodies within the glomeruli during proteinuria, suggesting antibody-independent mechanisms of B cell immunobiology is responsible. We hypothesise that B cell-derived factors are directly responsible for this phenotype, and this is currently being tested.

\title{
A38 A NOVEL MODEL OF MURINE B CELL-MEDIATED PROTEINURIA IDENTICAL TO HUMAN MINIMAL CHANGE DISEASE
}

A H J Kim, ${ }^{1}$ A S Shaw2, ${ }^{1}$ Division of Rheumatology, Department of Internal Medicine, Washington University School of Medicine, St Louis, MO 63110, USA; ${ }^{2}$ Division of Immunobiology, Department of Pathology and Immunology, Washington University School of Medicine, St Louis, MO 63110, USA; ${ }^{3}$ Howard Hughes Medical Institute, Washington University School of Medicine, St Louis, MO 63110, USA

\subsection{6/ard.2010.129585}

Minimal change disease remains the most common aetiology for paediatric nephrotic syndrome. The contributions of B cells to podocyte effacement and proteinuria remain unknown. We have developed a novel murine model of B-cell induced proteinuria with pathologic characteristics identical to human minimal change disease mediated through antibody-independent mechanisms. Hen egg lysozyme (HEL), once biotinylated and complexed to avidin, was found in the glomerular basement membrane (GBM) within 30 minutes following intravenous 